Originalien

Notfall Rettungsmed 2021 $\cdot 24: 134-142$ https://doi.org/10.1007/s10049-020-00695-7 Online publiziert: 27. März 2020

(c) Der/die Autor(en) 2020

J. C. Stephan ${ }^{1,2} \cdot$ T. Grossner $\cdot$ L. M. Stephan-Paulsen ${ }^{2,4} \cdot$ M. A. Weigand ${ }^{2} \cdot$

G. Schmidmaier ${ }^{3}$ E. Popp ${ }^{2}$

' Klinik für Anästhesiologie und Operative Intensivmedizin, Universitätsklinikum Schleswig-Holstein, Campus Kiel, Kiel, Deutschland

${ }^{2}$ Klinik für Anästhesiologie, Sektion Notfallmedizin, Universitätsklinikum Heidelberg, Heidelberg, Deutschland

${ }^{3}$ Zentrum für Orthopädie, Unfallchirurgie und Paraplegiologie, Klinik für Orthopädie und Unfallchirurgie, Universitätsklinikum Heidelberg, Heidelberg, Deutschland

${ }^{4}$ Zentrum für Innere Medizin, Klinik für Kardiologie, Pneumologie und Innere Medizin, imland Klinik Rendsburg, Rendsburg, Deutschland

\title{
Evaluation der
}

Aufnahmekriterien von Patienten nach Verkehrsunfall in den Schockraum

schwer verletzt eingestuft, 3377 Personen verstarben [1]. Unter den 71.109 tödlich oder schwer verletzten Personen befanden sich 30.575 Insassen von Personenkraftwagen (Pkw; [1]).

Patienten nach einem Verkehrsunfall werden im Rahmen des ersten medizinischen Kontakts vom Notarzt und Rettungsdienstpersonal untersucht und bezüglich ihrer Verletzungsschwere beurteilt. Die Deutsche Gesellschaft für Unfallchirurgie (DGU) gibt mit der S3Leitlinie Polytrauma/SchwerverletztenBehandlung [2] Handlungsempfehlungen für die präklinische Versorgung, klinische Abläufe im Schockraum sowie die erste Operationsphase von potenziell lebensgefährlich verletzten Patienten.

Schwer verletzte Patienten sollten in einem überregionalen Traumazentrum behandelt werden, da für diese Behandlungseinrichtungen ein verbessertes Überleben und ein verbessertes funktionelles Outcome beschrieben wurden. Zudem sind die Behandlungskosten im Vergleich mit einem nichtspezialisierten Krankenhaus geringer [3, 4]. Die primäre Aufnahmestelle für schwer verletzte Patienten im Traumazentrum ist der Schockraum. Als bereits präklinisch erfassbare Parameter für die Aufnahme in den Schockraum werden in der S3-
Leitlinie Polytrauma/SchwerverletztenBehandlung [2] zwei Gruppen von Kriterien genannt: Kriterien mit Empfehlungsgrad A („soll“) orientieren sich direkt am Zustand des Patienten, während Kriterien mit Empfehlungsgrad B („sollte“) isoliert das Unfallgeschehen beurteilen.

Die diesem Teil der Leitlinie zugrunde liegenden Publikationen stammen aus den Jahren 1988-2006, wobei die darin behandelten Daten bis in die 1970er-Jahre zurückreichen und somit fast 40 Jahre alt sind [2].

Die technische Weiterentwicklung von Fahrzeugkonstruktion und Sicherheitsausstattung sowie Änderungen von Gesetzen zur Verkehrssicherheit werden daher von der gültigen Leitlinie nicht abgebildet. Moderne Fahrzeuge (Durchschnittsalter Pkw in Deutschland am 01.01.2014 8,8 Jahre) mit Sicherheitsmerkmalen wie beispielsweise Airbags oder die Gurtanlagepflicht werden als protektive Faktoren gesehen und führen $\mathrm{zu}$ einer geringeren Verletzungsschwere sowie zu einer geringeren Sterblichkeit [5-7].

Für den Zeitraum 1991-2011 konnte bei einer Analyse von Verkehrsunfällen in Deutschland ein Rückgang der Verletzungsschwere sowie der Mortali- 


\section{Infobox 1 Kriterien mit dem Empfehlungsgrad A zur Aktivierung des Schockraumteams}

Kriterien mit dem Empfehlungsgrad A (nach [2]):

- Systolischer Blutdruck unter $90 \mathrm{~mm} \mathrm{Hg}$ (altersadaptiert bei Kindern) nach Trauma

- Vorliegen von penetrierenden Verletzungen der Rumpf-Hals-Region

- Vorliegen von Schussverletzungen der Rumpf-Hals-Region

- GCS unter 9 nach Trauma

- Atemstörungen/Intubationspflicht nach Trauma

- Frakturen von mehr als 2 proximalen Knochen

- Instabiler Thorax

- Beckenfrakturen

- Amputationsverletzung proximal der Hände/Füße

- Querschnittsverletzung

- Offene Schädelverletzungen

- Verbrennungen $>20 \%$ und Grad 2b

tät nachgewiesen werden. Im Vergleich der Untersuchungszeiträume 1991-1995 und 2006-2011 wurde ein Rückgang der Mortalität um 68,8\% (absolute Risikoreduktion: 1,6 auf $0,5 \%$ ) sowie ein Rückgang der Patienten mit Injury Severity Score (ISS) $\geq 16$ um $53,8 \%$ (2,6 auf $1,2 \%$ ) beschrieben [5]. Betrachtet man den Maximum Abbreviated Injury Score (MAIS) für die Vergleichszeiträume, so wurde ein Rückgang um 50,3\% für MAIS 2+ (maximaler AIS $\geq 2$ ) sowie um $65,4 \%$ für MAIS 4+ (maximaler AIS $\geq 4$ ) ermittelt. Der MAIS $<2$ (maximaler AIS $<2$ ) zeigt mit einer Zunahme um 8,5\% einen Anstieg der nicht oder leicht verletzten $\mathrm{Pa}$ tienten [5].

Folglich ist festzustellen, dass neben primär kritischen Patienten zunehmend auch Patienten ohne schwere oder lebensgefährliche Verletzungen dem Schockraum zugeführt werden, wodurch die absolute Zahl an Schockraumzuweisungen an einigen Zentren um bis zu $70 \%$ gestiegen ist [8]. Die von der DGU beschriebene Übertriage von 25 bis $35 \%$ [2] wird in der Realität wahrscheinlich deutlich überschritten. So werden in einer aktuellen Arbeit Raten an Übertriage, also unnötig in den Schockraum aufgenommenen Patienten, von bis zu $85 \%$ im internationalen Vergleich beschrieben [9].

\section{Polytrauma}

Da die Definition des Polytraumas seit Längerem auf internationaler Ebene kritisch diskutiert wird und eine einheitliche Definition nicht existiert, arbeitet seit 2012 eine internationale Arbeitsgruppe an einer neuen Begriffsbestimmung [10]. Im deutschsprachigen Raum hat sich seit Ende der 70er-Jahre die klinische Definition des Polytraumas von $\mathrm{H}$. Tscherne durchgesetzt [11] und wurde auch lange Zeit in dieser Form an den medizinische Hochschulen gelehrt. Nach Tscherne und Trentz liegt ein Polytrauma vor, wenn mindestens zwei Körperregionen verletzt sind, von denen eine oder deren Kombination lebensgefährlich ist [11]. Ebenfalls angewendet wird eine Definition unter Berücksichtigung eines ISS $\geq 17$ sowie der Dysfunktion von primär nicht verletzten Organen oder Organsystemen [12]. Im Weißbuch Schwerverletztenversorgung der DGU wird ein Schwerstverletzter anhand eines ISS $\geq 16$ definiert [13]. Die „Berlin-Definition“ des Polytraumas, welche einen ISS $>15$, einen AIS $\geq 3$ in mindestens zwei Körperregionen (entsprechend einem ISS $\geq 18$ ) sowie eine Über- bzw. Unterschreitung mindestens eines spezifischen Werts der fünf Kategorien Blutdruck, Bewusstsein, Azidose, Koagulopathie oder Alter voraussetzt, wurde erst vor Kurzem eingeführt und ist bereits Bestandteil der Auswertung im Traumaregister der DGU [14].

Gemäß internationalen Studien wird ein ISS $\geq 16$ als schwerwiegendes beziehungsweise schockraumrelevantes Trauma definiert [15-17]. Andere Arbeiten berücksichtigen ebenfalls eine intensivmedizinische Versorgung von $>24 \mathrm{~h}$, die Notwendigkeit einer Sofortoperation oder das Versterben während des Krankenhausaufenthalts [18, 19].

\section{Leitlinienkriterien}

Gemäß der S3-Leitlinie Polytrauma/ Schwerverletzten-Behandlung [2] der DGU „soll“ die Aktivierung des Schockraumteams erfolgen, sofern der Patient Verletzungen entsprechend den Kriterien mit Empfehlungsgrad A aufweist (siehe - Infobox 1).
Aufgrund der Kinematik des Unfallhergangs „sollte“ die Aktivierung des Schockraumteams erfolgen, sofern der Patient ein Kriterium mit Empfehlungsgrad B, wie beispielsweise einen „Verkehrsunfall (VU) mit einer Geschwindigkeitsveränderung von delta $>30 \mathrm{~km} / \mathrm{h}^{\text {“ }}$ aufweist [2]. Die Leitlinie zeigt nicht auf, auf welchen Erkenntnissen vor Ort dieses Kriterium evaluiert werden soll. Es muss daher davon ausgegangen werden, dass in Unkenntnis von Ausgangsgeschwindigkeit, Kollisionsgeschwindigkeit und vektorieller Geschwindigkeitsänderung die Einschätzung subjektiv und potenziell ungenau ist.

\section{Nachteile einer liberalen Indikation für die Schockraum- aufnahme}

Für eine optimale Patientenversorgung im Schockraum sind ein hoher Personalschlüssel und eine große Ressourcenvorhaltung notwendig. Routineabläufe verschiedener Klinikabteilungen müssen daher intermittierend zugunsten der über den Schockraum aufgenommenen Patienten ab- oder zumindest unterbrochen werden.

Patienten und Personal werden durch die in der S3-Leitlinie bei Hochrasanztraumata (u.a. zum Ausschluss eines schweren Thoraxtraumas bei „Verkehrsunfall Vmax $>50 \mathrm{~km} / \mathrm{h}^{\prime}$ ) empfohlenen Röntgen- und CT-Untersuchungen ionisierender Strahlung ausgesetzt [2]. In der Frühphase der Diagnostik spielt laut aktueller Literatur die Computertomographie bei schwer verletzten Patienten eine wichtige Rolle [20]. Zwar sind die Alarmierungskriterien für den Schockraum in der Leitlinie nicht komplett deckungsgleich mit der Indikation zur CT, da aber die Kriterien Schätzwerte („Geschwindigkeitsänderung von delta $>30 \mathrm{~km} / \mathrm{h}^{\text {“ }}$ und „Verkehrsunfall Vmax $>50 \mathrm{~km} / \mathrm{h}^{\text {“) }}$ enthalten, begünstigt die liberale Schockraumaufnahme die Anwendung der CT. Eine Ganzkörper-CTUntersuchung senkt bei potenziell lebensbedrohlich verletzten Patienten die Mortalitätsrate um 13-25\% [21, 22], jedoch wirken bei einer Traumaspirale $10-20 \mathrm{mSv}$ auf den Patienten ein, was eine strahleninduzierte Mortalität von 
Notfall Rettungsmed 2021 · 24:134-142 https://doi.org/10.1007/s10049-020-00695-7

(c) Der/die Autor(en) 2020

\section{J. C. Stephan · T. Grossner · L. M. Stephan-Paulsen · M. A. Weigand · G. Schmidmaier · E. Popp}

\section{Evaluation der Aufnahmekriterien von Patienten nach Verkehrsunfall in den Schockraum}

\section{Zusammenfassung}

Hintergrund. Die aktuelle S3-Leitlinie Polytrauma/Schwerverletzten-Behandlung der Deutschen Gesellschaft für Unfallchirurgie (DGU) empfiehlt bei einem Pkw-Unfall mit einer Geschwindigkeitsveränderung von delta $>30 \mathrm{~km} / \mathrm{h}$ die Versorgung in einem Schockraum ohne Berücksichtigung der Verletzungen des Patienten. Ziel dieser Studie war es zu untersuchen, ob Patienten, die ausschließlich aufgrund dieses Kriteriums über einen Schockraum aufgenommen wurden, relevante Verletzungen aufwiesen, die intensivmedizinische Behandlungen oder (Not-)Operationen benötigten.

Methode. Nach einem Pkw-Unfall wurden Patienten ohne spezifische Verletzung, bei denen ausschließlich eine Geschwindigkeitsveränderung von delta $>30 \mathrm{~km} / \mathrm{h}$ vorlag (Empfehlungsgrad B der S3-Leitlinie), der Studiengruppe, Patienten mit Verletzungen gem. Empfehlungsgrad A der Leitlinie der Vergleichsgruppe zugeordnet. Ein schockraumrelevantes Trauma wurde als
Injury Severity Score (ISS) $\geq 16$, operative Versorgung innerhalb $24 \mathrm{~h}$, intensivmedizinische Überwachung $>24 \mathrm{~h}$, Versterben während des Krankenhausaufenthalts sowie DGU-Basiskollektiv (MAIS3+ oder MAIS2 mit Intensivverweildauer $>24 \mathrm{~h}$ bzw. Versterben während des Krankenhausaufenthalts) definiert.

Ergebnisse. Der Vergleich zeigte einen hochsignifikanten Unterschied in Bezug auf den mittleren ISS $(p \leq 0,001)$, ein schockraumrelevantes Trauma (ISS $\geq 16 ; p \leq 0,001$ ), eine intensivmedizinische Versorgung $>24 \mathrm{~h}(p \leq 0,001)$, Operation innerhalb von $24 \mathrm{~h}$ nach Krankenhausaufnahme $(p \leq 0,001)$, Letalität $(p \leq 0,001)$ sowie DGUBasiskollektiv $(p \leq 0,001)$. Anhand dieser Ergebnisse konnte gezeigt werden, dass innerhalb der Studiengruppe (Geschwindigkeitsveränderung von delta $>30 \mathrm{~km} / \mathrm{h}$; Empfehlungsgrad B der S3-Leitlinie) lediglich ein Patient eine Traumafolge aufwies, die eine intensivmedizinische Behandlung
$>24$ h oder eine Operation nötig machte. Studien- und Vergleichsgruppen waren in Bezug auf das mittlere Alter $(p=1,778)$, das männliche Geschlecht $(p=0,1728)$ sowie die durchschnittliche Unfallgeschwindigkeit $(p=0,4606)$ vergleichbar.

Diskussion. Ein alleiniges Vorliegen eines Pkw-Unfalls mit einer Geschwindigkeitsveränderung von delta $>30 \mathrm{~km} / \mathrm{h}$ kann nicht als adäquater Prädiktor für ein schockraumrelevantes Trauma gesehen werden. Weitere Studien könnten durch eine Leitlinienanpassung eine weiterhin sichere und hochwertige Patientenversorgung bei Reduktion von personellen und finanziellen Belastungen ermöglichen.

\section{Schlüsselwörter}

Schockraumindikation · Schockraumalarmierung - Schwerverletztenversorgung - Trauma . Unfall

\section{Evaluation of trauma centre admission criteria for patients involved in road traffic accidents}

\section{Abstract}

Background. The current S3 guideline of the German Trauma Society (Deutsche Gesellschaft für Unfallchirurgie, DGU) recommends that all patients involved in road traffic accidents with a change of velocity $>30 \mathrm{~km} / \mathrm{h}$ be treated in an emergency room irrespective of their injures. Methods. Following road traffic accidents, patients without guideline-specific injuries were assigned to the study group, while patients with injuries according to a level-A recommendation were assigned to the reference group. Emergency room-relevant trauma was defined as an injury severity score (ISS) $\geq 16$, surgical treatment within $24 \mathrm{~h}$, intensive care $>24 \mathrm{~h}$, in-hospital death and
DGU base collective (maximum abbreviated injury score [MAIS] 3+ or MAIS2 with intensive care $>24 \mathrm{~h}$ or in-hospital death).

Results. The comparison showed a highly significant difference concerning average ISS ( $p \leq 0.001)$, emergency room-relevant trauma (ISS $\geq 16: p \leq 0.001$ ), intensive care $>24 \mathrm{~h}(p \leq 0.001)$, surgical treatment within $24 \mathrm{~h}(p \leq 0.001)$, death $(p \leq 0.001)$ and DGU base collective $(p \leq 0.001)$. The results showed that only one patient in the study group (change of velocity $>30 \mathrm{~km} / \mathrm{h}$, according to the $S 3$ guideline) required $>24 \mathrm{~h}$ intensive care treatment. Study and reference groups were comparable concerning average age $(p=1.778)$, male sex $(p=0.1728)$ and average accident speed $(p=0.4606)$.

Conclusion. A simple road traffic accident with a change of velocity $>30 \mathrm{~km} / \mathrm{h}$ is not an appropriate predictor of emergency roomrelevant trauma. By adjusting the guideline, further surveys could ensure safe and highquality treatment while reducing personnel and financial burdens.

\section{Keywords}

Emergency room indication - Emergency room activation - Serious injury management . Trauma. Accident
1:1250 für einen Erwachsenen mittleren Alters ergibt [22]. Eine Ganzkörper-CT mit einer nicht bewerteten Dosisleistung von 14 bis $21 \mathrm{mGy}$ steigert das Lebenszeitrisiko, an Krebs zu erkranken, um $1,9 \%$ [23].

Bei der Versorgung eines nicht schwer verletzten Patienten in einem Haus der Maximalversorgung entstehen bei gleichwertiger Versorgung deutlich hö- here Kosten als bei der Behandlung in einem kleineren Krankenhaus, welche 2013 mit durchschnittlich 5590 US-\$ pro Behandlungsfall beziffert wurden [24].

\section{Methodik}

\section{Patienteneinschluss}

Das analysierte Patientenkollektiv umfasste potenziell schwer verletzte Patienten, welche nach dem Kriterium „Verkehrsunfall (VU) mit einer Geschwindigkeitsveränderung von delta $>30 \mathrm{~km} / \mathrm{h}^{\text {“ }}$ (als Insasse eines Pkw oder Lkw) der S3- 
Leitlinie über den Schockraum der Chirurgischen Klinik des Universitätsklinikums Heidelberg aufgenommen beziehungsweise dort versorgt wurden und ein Mindestalter von 16 Jahren aufwiesen.

Die Studiengruppe bildeten Patienten, welche ausschließlich das eine B-Kriterium „Geschwindigkeitsveränderung von delta $>30 \mathrm{~km} / \mathrm{h}$ “ aufwiesen.

Patienten der Vergleichsgruppe wiesen zusätzlich Verletzungen oder Veränderungen der Vitalparameter entsprechend einem oder mehreren Kriterien mit Empfehlungsgrad A auf.

Die Studie umfasste den Zeitraum vom 01.01.2014, 0:00 Uhr, bis zum 31.12.2014, 23:59 Uhr.

Ein positives Votum der Ethikkommission lag unter der Vorgangsnummer S-585/2014 vor.

\section{Datenerhebung}

Zur Beurteilung des tatsächlichen klinischen Zustands der Patienten wurde die Verletzungsschwere anhand des ISS nach Abschluss der stationären Versorgung ermittelt. Die Datenerhebung sowie Berechnung des ISS erfolgten ausschließlich durch drei Mitarbeiter der Arbeitsgruppe Schockraum unter Verwendung aller zur Verfügung stehenden Dokumentation und Befunde. Die Ergebnisse wurden stichprobenartig gegenseitig überprüft.

Zusätzlich wurden im Rahmen der Studie die Dauer der intensivmedizinischen Überwachung, Notwendigkeit einer Notoperation, Notwendigkeit einer frühelektiven Operation (innerhalb von $24 \mathrm{~h}$ nach Aufnahme), Notwendigkeit invasiver Versorgung im Schockraum, Tod während des Krankenhausaufenthalts sowie Zuordnung in das DGUBasiskollektiv (MAIS 3+ oder MAIS 2 mit Intensivverweildauer $>24 \mathrm{~h}$ bzw. Versterben während des Krankenhausaufenthalts) erfasst und ausgewertet.

\section{Studiendesign}

Die Studie wurde als eine prospektive, explorative, monozentrische und deskriptive Registerstudie im Sinne einer Pilotstudie durchgeführt. Die Daten der Patienten wurden nach Aufnahme in den Schockraum der Chirurgischen Klinik des Universitätsklinikums Heidelberg erfasst. Betrachtet wurden alle aufgenommenen Patienten. Es wurde keine Randomisierung durchgeführt, die Therapie aller Patienten fand nach den gleichen Algorithmen statt. Es fand kein Follow-up statt. Alle relevanten Daten wurden während des Krankenhausaufenthalts der $\mathrm{Pa}$ tienten gewonnen.

Die Auswertung bestehender Register, wie beispielsweise des TraumaRegisters $\mathrm{DGU}^{\circledR}$, erschien den Untersuchern dieser Studie als nicht ausreichend, da in dem Register nur Fälle ab einer bestimmten Verletzungsschwere erfasst beziehungsweise unverletzte Patienten nicht vollständig registriert werden. Ebenso ist dort die Detailtiefe nicht gegeben, welche im Rahmen dieser Studie angewendet wurde.

\section{Statistische Methoden}

Aufgrund des Studiendesigns wurde der Einschlusszeitraum von einem Kalenderjahr festgesetzt. Eine Fallzahlberechnung wurde nicht durchgeführt.

Relevante Eingangs- und Zielparameter wurden deskriptiv und vergleichend analysiert. Stetige Daten wurden mit Minimum, Maximum, Mittelwert, Median und Standardabweichung beschrieben. Kategoriale Daten wurden mit absoluter und relativer Häufigkeit beschrieben.

Die statistische Auswertung erfolgte mit Chi-Quadrat-Test, ungepaartem t-Test, Mann-Whitney-U-Test, logistischen Regressionsanalysen und varianzanalytischen Methoden. Das Signifikanzniveau wurde mit $p \leq 0,001$ festgesetzt. $p$-Werte, die sich aus diesen statistischen Analysen ergeben, sind rein deskriptiv und haben keinen konfirmatorischen Wert.

\section{Ergebnisse}

\section{Patientenkollektiv}

Im Studienzeitraum wurden 808 Patienten im Schockraum der Chirurgischen Klinik des Universitätsklinikums Heidelberg versorgt. 238 Patienten (29,5\%) wiesen einen Pkw- oder Lkw-Unfall auf. 197 Patienten $(82,7 \%$ ) wurden in die Studiengruppe, 28 Patienten $(11,8 \%)$ in die
Vergleichsgruppe eingeschlossen; $13 \mathrm{~Pa}$ tienten $(5,5 \%)$ wurden aufgrund des $\mathrm{Al}$ ters unter 16 Jahren ausgeschlossen.

Das Alter der 197 Patienten der Studiengruppe reichte von 17 bis 85 $(42,2 \pm 18,6)$ Jahren. Bei den 28 Patienten der Vergleichsgruppe reichte das Alter von 17 bis $78(47,4 \pm 21,5)$ Jahren. Die Verteilung ist mit $p=0,18$ nicht signifikant und wird als vergleichbar betrachtet.

Männliches Geschlecht wiesen $114 \mathrm{~Pa}$ tienten (58\%) der Studiengruppe und 20 Patienten $(71 \%)$ der Vergleichsgruppe auf, die Verteilung ist mit $p=0,17$ nicht signifikant. Die durchschnittliche Unfallgeschwindigkeit wurde in der Studiengruppe mit $78 \pm 29 \mathrm{~km} / \mathrm{h}$, in der Vergleichsgruppe mit $84 \pm 26 \mathrm{~km} / \mathrm{h}$ angegeben, der Unterschied ist mit $p=0,4606$ nicht signifikant.

\section{Schockraumindikation nach Pkw-Unfall}

Alle 197 Patienten der Studiengruppe sowie alle 28 Patienten der Vergleichsgruppe wiesen das vorgegebene Einschlusskriterium „VU mit Geschwindigkeitsveränderung von delta $>30 \mathrm{~km} / \mathrm{h}^{\text {“ }}$ auf. Die Angabe zur Geschwindigkeitsänderung beruhte auf der Aussage des behandelnden Notarztes.

\section{Verletzungsschwere anhand ISS}

Der bei den 197 Studienpatienten ermittelte ISS lag zwischen 0 und $18(1,8 \pm 3,0$; $\mathrm{Q} 1=0 ; \mathrm{Q} 3=2$; Median =1). In der Vergleichsgruppe wurde ein ISS zwischen 0 und 75 (20,9 $\pm 15,3 ; \mathrm{Q} 1=9 ; \mathrm{Q} 3=27,5$; Median $=17$ ) errechnet. Der Unterschied des mittleren ISS war mit $p \leq 0,001$ hochsignifikant. Die Verteilung des ISS ist in

- Abb. 1 dargestellt.

Ein schockraumrelevantes Trauma, definiert über den ISS $\geq 16$, lag in der Studiengruppe bei einem Patienten (0,5\%), in der Studiengruppe bei 17 Patienten $(60,7 \%)$ vor. Der Unterschied war mit $p \leq 0,001$ ebenfalls hochsignifikant.

\section{Intensivverweildauer}

In der Studiengruppe wurden 189 von 197 Patienten (95,9\%) nach Abschluss 


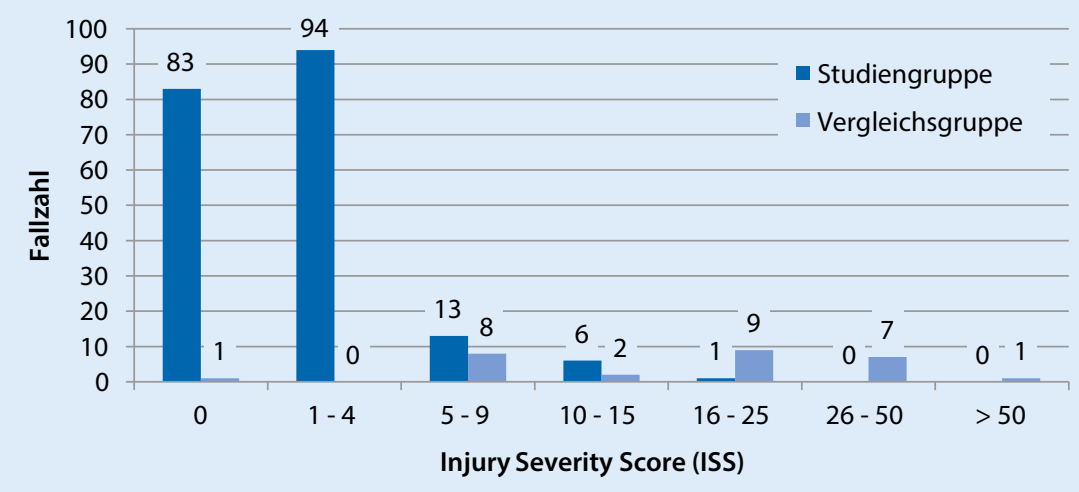

Abb. $1 \Delta$ Verteilung des Injury Severity Score in Studien- und Vergleichsgruppe

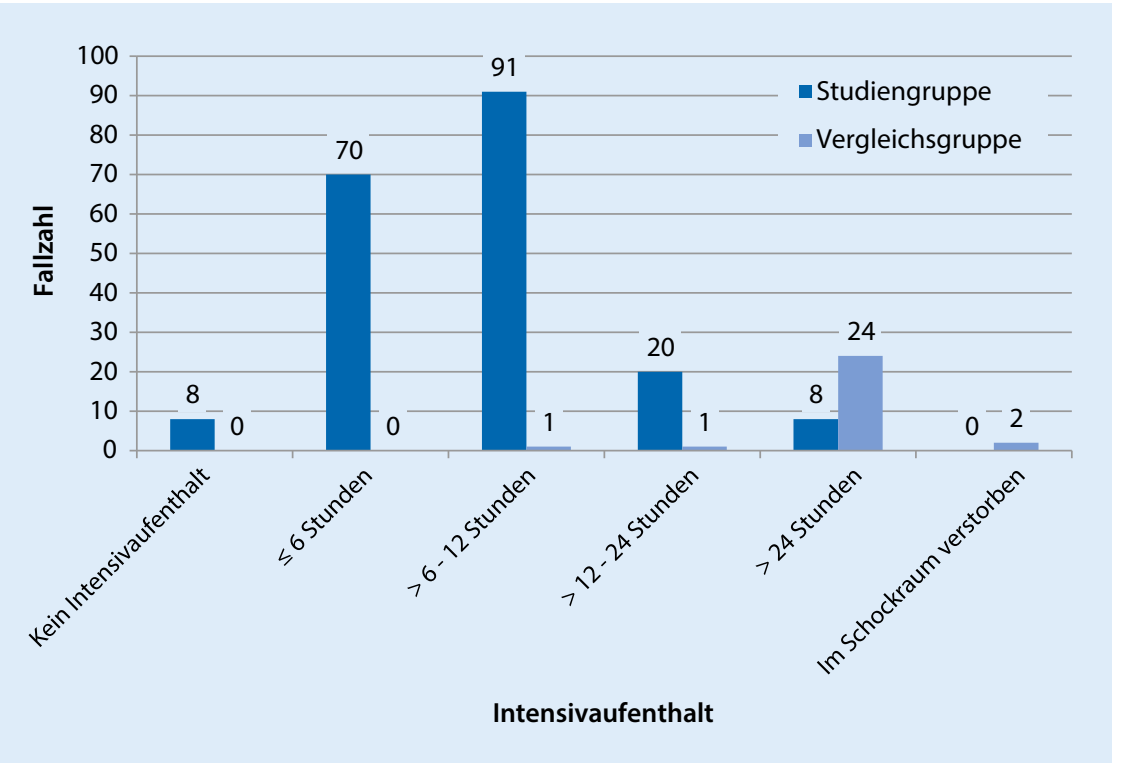

Abb. 2 \ Verteilung der Verweildauer auf einer Intensivstation in Studien- und Vergleichsgruppe

der Versorgung im Schockraum zur Überwachung auf eine Intensivstation, Intermediate Care Unit oder in einen Aufwachraum mit vollständiger intensivmedizinischer Ausstattung übernommen. Die Verweildauer betrug 1-168 $(11,4 \pm 15,8)$ Stunden. 8 von 197 Patienten $(4,1 \%)$ wurden $>24 \mathrm{~h}$ intensivmedizinisch überwacht. 62 der 189 Patienten $(32,8 \%)$ wurden von der Intensivstation nach Hause entlassen. Bei 97 von $197 \mathrm{~Pa}$ tienten $(49,2 \%)$ war eine Entlassung aus der stationären Behandlung innerhalb von $24 \mathrm{~h}$ möglich.

In der Vergleichsgruppe wurden 26 von 28 Patienten $(92,9 \%)$ auf eine Intensivstation aufgenommen. 2 von $28 \mathrm{~Pa}$ tienten $(7,1 \%)$ verstarben im Schockraum. Die Verweildauer der Patienten auf einer Intensivstation betrug 9-1320 $(334 \pm 377)$ Stunden (0,4bis 55 [14 $\pm 15,7]$ Tage). 24 von 28 Patienten (85,7\%) wurden $>24$ h intensivmedizinisch überwacht. Keiner der 28 Patienten wurde innerhalb von $24 \mathrm{~h}$ aus der stationären Behandlung entlassen.

Die Analyse der Verweildauer auf einer Intensivstation ist mit $p \leq 0,001$ hochsignifikant länger als in der Studiengruppe.

Die Verteilung der Verweildauer im Intensivbereich ist in $\bullet \mathbf{A b b} .2$ dargestellt.

\section{Notwendigkeit einer Not- oder frühelektiven Operation}

Bei 3 von 197 Patienten der Studiengruppe $(1,5 \%)$ wurde innerhalb der ersten
$24 \mathrm{~h}$ ein operativer Eingriff, jedoch keine lebensrettende Notoperation, durchgeführt. In der Vergleichsgruppe wurde bei 14 von 28 Patienten (50\%) innerhalb der ersten $24 \mathrm{~h}$ ein operativer Eingriff durchgeführt, 71,4\% der Eingriffe als Notoperation. Die Studiengruppe unterscheidet sich gegenüber der Vergleichsgruppe, sowohl bei Notoperationen mit $p \leq 0,001$ als auch bei frühelektiven Operationen mit $p \leq 0,001$, hochsignifikant.

\section{Invasive Versorgung im Schockraum}

In der Studiengruppe wurde bei keinem von 197 Patienten (0\%) im Schockraum eine invasive Versorgung durchgeführt. In der Vergleichsgruppe wurde bei 8 von 28 Patienten $(28,6 \%)$ eine invasive Versorgung im Schockraum notwendig. Hierbei handelte es sich um Intubation (3,6\%), ZVK-Anlage (10,7\%), Thoraxdrainage $(14,3 \%)$ sowie Reanimation $(7,1 \%)$. Der Unterschied ist mit $p \leq 0,001$ hochsignifikant.

\section{Letalität}

In der Studiengruppe verstarb kein $\mathrm{Pa}$ tient $(0 \%)$ während der Behandlung im Schockraum sowie während des stationären Aufenthalts am Universitätsklinikum Heidelberg. In der Vergleichsgruppe verstarben 2 von 28 Patienten $(7,1 \%)$ im Schockraum und ein Patient (3,6\%) während des stationären Aufenthalts. Somit ergibt sich bei einer Letalität von $0 \%$ vs. $10,7 \%$ mit $p \leq 0,001$ ein hochsignifikanter Unterschied zwischen den Gruppen.

\section{DGU-Basiskollektiv}

Die Deutsche Gesellschaft für Unfallchirurgie definierte 2015 das sog. Basiskollektiv anhand der Kriterien MAIS3+ oder MAIS2 mit Intensivverweildauer $>24 \mathrm{~h}$ bzw. Versterben während des Krankenhausaufenthalts [25]. In der Studiengruppe erfüllten 13 Patienten $(6,6 \%)$ die Kriterien für das Basiskollektiv, in der Vergleichsgruppe waren es 27 Patienten (96,4\%). Der Unterschied ist mit $p \leq 0,001$ hochsignifikant. 


\begin{tabular}{|c|c|c|c|c|c|c|c|c|}
\hline & $\begin{array}{l}\text { Studien- } \\
\text { gruppe }\end{array}$ & $\begin{array}{l}\text { Kriterium } \\
\text { erfüllt }\end{array}$ & $\begin{array}{l}\text { Anteil } \\
(\%)\end{array}$ & $\begin{array}{l}\text { Übertriage } \\
\text { (\%) }\end{array}$ & $\begin{array}{l}\text { Vergleichs- } \\
\text { gruppe }\end{array}$ & $\begin{array}{l}\text { Kriterium } \\
\text { erfüllt }\end{array}$ & $\begin{array}{l}\text { Anteil } \\
(\%)\end{array}$ & $\begin{array}{l}\text { Übertriage } \\
\text { (\%) }\end{array}$ \\
\hline $\mathrm{ISS} \geq 16$ & 197 & 1 & 0,5 & 99,5 & 28 & 17 & 60,7 & 39,3 \\
\hline $\begin{array}{l}\text { Intensivmedizinische Überwa- } \\
\text { chung }>24 \mathrm{~h}\end{array}$ & 197 & 8 & 4,1 & 95,9 & 28 & 24 & 85,7 & 14,3 \\
\hline $\begin{array}{l}\text { ISS } \geq 16 \text { und/oder intensivmedizi- } \\
\text { nische Überwachung }>24 \mathrm{~h}\end{array}$ & 197 & 8 & 4,1 & 95,9 & 28 & 26 & 92,9 & 7,1 \\
\hline Not- oder frühelektive Operation & 197 & 3 & 1,5 & 98,5 & 28 & 14 & 50,0 & 50,0 \\
\hline $\begin{array}{l}\text { Invasive Versorgung im Schock- } \\
\text { raum }\end{array}$ & 197 & 0 & 0,0 & 100,0 & 28 & 8 & 28,6 & 71,4 \\
\hline Letalität & 197 & 0 & 0,0 & 100,0 & 28 & 3 & 10,7 & 89,3 \\
\hline DGU-Basiskollektiv & 197 & 13 & 6,6 & 93,4 & 28 & 27 & 96,4 & 3,6 \\
\hline
\end{tabular}

Tab. 2 Übertriage innerhalb von Geschwindigkeitsgruppen bezogen auf das Gesamtkollektiv

\begin{tabular}{|l|l|l|l}
\hline & Fallzahl & ISS $\geq \mathbf{1 6}$ & $\begin{array}{l}\text { Übertriage } \\
\text { (\%) }\end{array}$ \\
\hline $30-50 \mathrm{~km} / \mathrm{h}$ & 38 & 0 & 100 \\
\hline $51-70 \mathrm{~km} / \mathrm{h}$ & 65 & 3 & 95,4 \\
\hline $71-100 \mathrm{~km} / \mathrm{h}$ & 49 & 4 & 91,8 \\
\hline $101-140 \mathrm{~km} / \mathrm{h}$ & 18 & 0 & 100 \\
\hline$>140 \mathrm{~km} / \mathrm{h}$ & 6 & 1 & 83,3 \\
\hline Unbekannt, aber vor Ort mit $>30 \mathrm{~km} / \mathrm{h}$ angenommen & 49 & 10 & 79,6 \\
\hline Mit Geschwindigkeitsangabe & 176 & 8 & 95,5 \\
\hline Alle Patienten & 225 & 18 & 92,0
\end{tabular}

\section{Übertriage nach einem Pkw-/Lkw-Unfall}

Übertriage beschreibt einen Zustand, bei dem nicht oder nur leicht verletzte $\mathrm{Pa}$ tienten wie schwer verletzte Patienten behandelt werden. Diese unnötige Versorgung zeigt sich beispielsweise durch die Aufnahme über einen Schockraum. Übertriage ist notwendig, um das Übersehen primär unkritischer Patienten $\mathrm{zu}$ verhindern $[2,9]$.

Bei Betrachtung der Übertriage in der Studien- sowie der Vergleichsgruppe bezüglich der ausgewerteten Kriterien zeigt sich ein deutlicher Unterschied, welcher in - Tab. 1 dargestellt wird.

\section{Übertriage in Abhängigkeit von der Unfallgeschwindigkeit}

Bei Betrachtung der geschätzten absoluten Unfallgeschwindigkeit wurden 207 von 225 Patienten (92\%) übertriagiert. Ein Zusammenhang zwischen der Unfallgeschwindigkeit und der Rate an Übertriage konnte nicht festgestellt werden.
Die Raten an Übertriage anhand der Unfallgeschwindigkeit sind in - Tab. 2 dargestellt.

\section{Diskussion}

Die Ergebnisse dieser Studie legen nahe, dass viele Patienten aufgrund eines Verkehrsunfalls unnötig der Schockraumversorgung zugeführt werden. Die Einschätzung des Notarztes, ob das Kriterium „Verkehrsunfall mit einer Geschwindigkeitsänderung von delta $>30 \mathrm{~km} / \mathrm{h}$ “ vorgelegen hat, konnte nicht als Prädiktor für eine schwere Verletzung, Notwendigkeit einer intensivmedizinischen Versorgung $>24$ h, Notwendigkeit einer Notoperation oder erhöhte Letalität nachgewiesen werden. Es resultiert eine hohe Rate an Übertriage mit entsprechend hohem personellem und finanziellem Aufwand sowie unnötiger bildgebender Diagnostik.

In dieser Studie wurde für einen PkwUnfall mit einer „Geschwindigkeitsver-

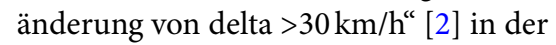
Studiengruppe eine Übertriage zwischen 93,4\% für das DGU-Basiskollektiv und
$99,5 \%$ bezogen auf einen ISS $\geq 16$ ermittelt.

Die Aussagekraft sowie der Nutzen von kinetischen Unfallkriterien werden in der gängigen Literatur, einschließlich der S3-Leitlinie Polytrauma/ Schwerverletzten-Behandlung selbst, kontrovers diskutiert. Der alleinige Unfallmechanismus oder die vermutete Unfallgeschwindigkeit konnte von keinem Autor als verlässlicher Prädiktor für die Verletzungsschwere der Patienten, eine verlängerte Intensiv- oder Krankenhausverweildauer beziehungsweise die Notwendigkeit von Operationen nachgewiesen werden [26, 27]. Die Kriterien des Unfallmechanismus werden für einen signifikanten Anteil der Übertriage verantwortlich gemacht [18]. Begründend hierfür findet sich der veraltete Wissenstand, welcher der Leitlinie zugrunde liegt. Durch die technische Weiterentwicklung der Automobile (Fahrzeugkonstruktion, Sicherheitsausstattung und angepasste Gesetze) lässt sich eine Zunahme der Übertriage postulieren, da protektive Faktoren, wie die Nutzung eines Sicherheitsgurtes (OR 0,41; CI $95 \%$ 0,32-0,49), das Vorhandensein von Airbags (OR 0,86; CI $95 \%$ 0,75-0,99) sowie modernere Fahrzeuge (OR 0,94; CI $95 \%$ 0,89-0,99 pro 5 Jahre Fahrzeugalter; [5]) in der aktuellen S3Leitlinie nicht berücksichtigt sind.

Bei der Auswertung von Unfalldaten in Deutschland konnten Ernstberger et al. einen Rückgang der Anzahl schwer verletzter Patienten sowie der Mortalität nachweisen [5]. Diese Ergebnisse decken sich mit den Beobachtungen dieser Studie. 


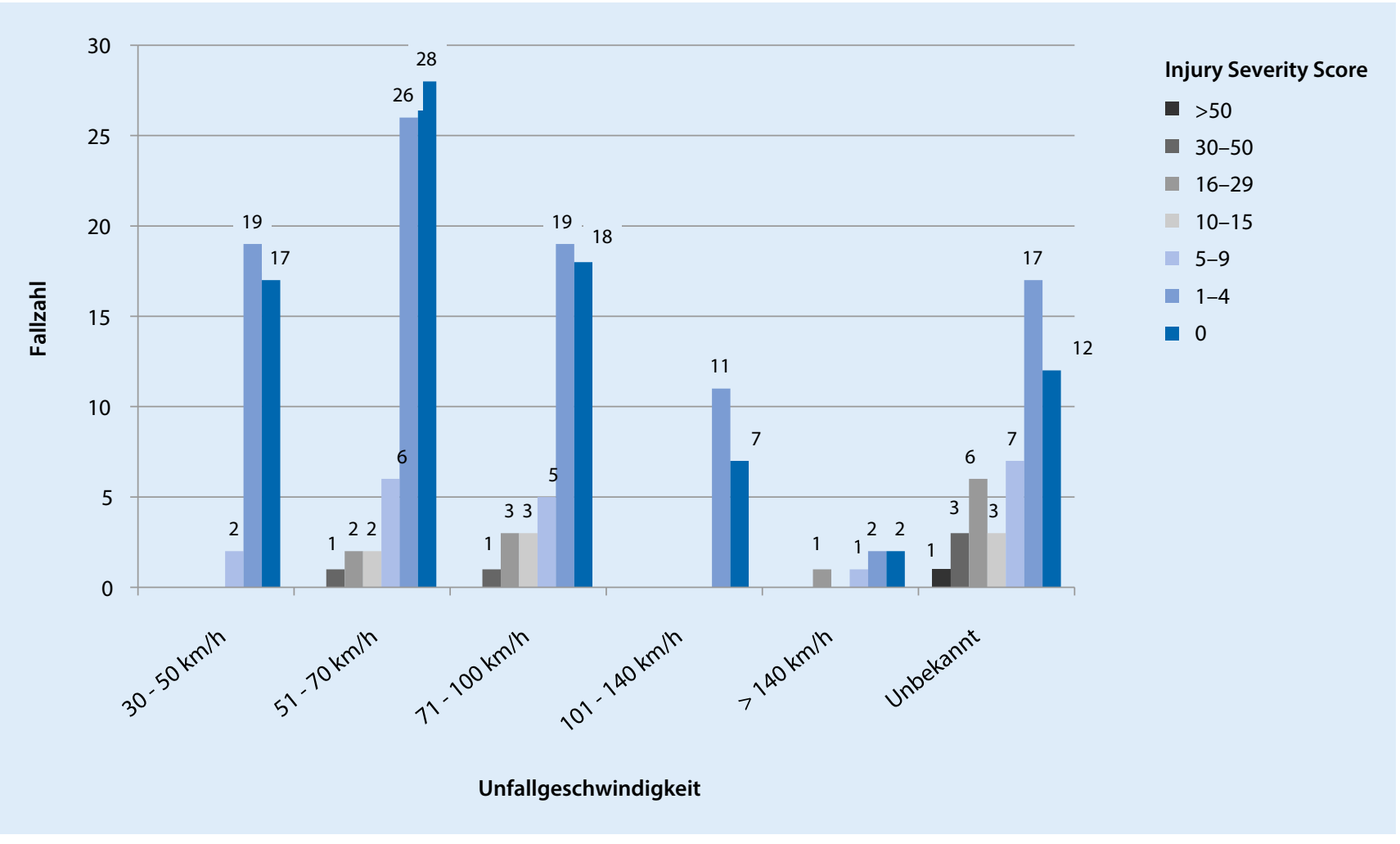

Abb. $3 \Delta$ Verletzungsschwere in Abhängigkeit von der Unfallgeschwindigkeit

Lerner et al. beschrieben 2011, dass bei lediglich 9\% der Patienten anhand des Unfallmechanismus eine adäquate Vorhersage der Verletzungsschwere möglich sei [19]. Bei 4,1 \% der Studiengruppenpatienten konnte ein entsprechendes Trauma bzw. eine intensivmedizinische Versorgungszeit nachgewiesen werden, was diese Ergebnisse bestätigt.

Bereits 2003 wurde gezeigte, dass lediglich $8 \%$ der Patienten mit unfallkriterienbedingter Aufnahme einen ISS $\geq 15$ aufwiesen [17]. In dieser Studie wiesen nur 0,5\% der Patienten der Studiengruppe ein entsprechendes Trauma auf.

Spering et al. konnten eine Verbesserung der Patientenversorgung sowie eine Reduktion der Über- und Untertriage dadurch erzielen, dass ein Notarzt am Unfallort die Patienten triagierte und einer strukturierten Schockraumversorgung zuführte, anstatt die Indikation starr anhand der DGU-Kriterien zu stellen [28].

Angenommene Unfallgeschwindigkeiten weichen häufig deutlich von den durch einen Unfallsachverständigen ermittelten Geschwindigkeiten ab [29].
Auch ist entscheidend, ob die Geschwindigkeitsveränderung innerhalb einer kurzen Zeit (Aufprall auf eine Stahlbetonwand) oder über einen längeren Zeitraum (Auffahrunfall auf rollendes Fahrzeug am Stauende oder longitudinale Leitplankenkollision) erfolgt. Bei einem Frontalzusammenstoß kann die Aufprallenergie leichter über die Karosserie („Knautschzone“) aufgenommen werden als bei einem Seitenaufprall, welcher hierdurch eine höhere Gefahr für den Fahrzeuginsassen aufweist.

Bei Betrachtung aller eingeschlossenen Patienten konnte kein Zusammenhang zwischen der Unfallgeschwindigkeit und der Verletzungsschwere festgestellt werden. In der Geschwindigkeitsgruppe bis $50 \mathrm{~km} / \mathrm{h}$ wurden 38 Patienten erfasst, der maximale ermittelte ISS betrug hier 9. Eine Unfallgeschwindigkeit über $100 \mathrm{~km} / \mathrm{h}$ wurde in 24 Fällen angegeben, hiervon wies jedoch lediglich 1 Patient $(4 \% ; 160 \mathrm{~km} / \mathrm{h})$ einen ISS von 18 auf. Besonders hervorzuheben ist, dass nur bei 8 von 176 Patienten $(4,6 \%)$ mit bekannter Unfallgeschwindigkeit ein ISS von $\geq 16$ vorlag. Hierbei war lediglich einer von 8 Patienten (12,5\%) schneller als $100 \mathrm{~km} / \mathrm{h}$; die andern 7 Patienten (87,5\%) wiesen Unfallgeschwindigkeiten zwischen 70 und $100 \mathrm{~km} / \mathrm{h}$ auf (• Abb. 3).

Schon 2006 wurde in den TriageGuidelines der amerikanischen Centers for Disease Control and Prevention das Kriterium der Unfallgeschwindigkeit als Prädiktor für schwerwiegende Verletzungen gestrichen, welches zuvor mit $>40 \mathrm{mph}$ (ca. $64 \mathrm{~km} / \mathrm{h}$ ) angegeben wurde [30]. Bei Verkehrsunfällen mit einer Unfallgeschwindigkeit von über $60 \mathrm{~km} / \mathrm{h}$ wurde 2008 eine Sensitivität von $44 \%$ bei einer Spezifität von $55 \%$ zur Identifikation von potenziell schwer verletzten Patienten angegeben [31]. Bereits 1996 wurde beschrieben, dass Autounfälle mit hoher Geschwindigkeit alleine nicht für die Identifikation von Patienten mit erhöhter Letalität, verlängertem Intensivaufenthalt oder Operationsindikation genutzt werden können [26].

Die in dieser Studie festgestellten hochsignifikanten Unterschiede in Bezug auf die Verletzungsschwere, intensivmedizinische Versorgungszeit, Notwendigkeit von Notoperationen oder invasiver 
Versorgung und nicht zuletzt die Letalität zeigen einen deutlichen Handlungsbedarf bezüglich der Indikationsstellung für die Schockraumversorgung. Wie in der Diskussion beschrieben, weisen internationale Veröffentlichungen der letzten Dekaden vergleichbare Ergebnisse auf.

In aktuellen Studien wird versucht, anhand eines überarbeiteten Kriterienkatalogs einen neuen Goldstandard für die Alarmierung des Schockraumteams $\mathrm{zu}$ entwickeln [9].

Durch adäquate Zuordnung der $\mathrm{Pa}$ tienten wären bei gleichbleibend hoher und sicherer Versorgungsqualität eine deutliche Entlastung des Personals, verbesserte Abläufe der Krankenhausroutine sowie eine Optimierung der Versorgungskosten möglich.

\section{Limitationen}

- Es konnten statistisch nur Patienten erfasst werden, welche nach einem Unfall im Schockraum des Universitätsklinikums versorgt wurden. Es ist von einer nicht unerheblichen Dunkelziffer unverletzter Personen auszugehen, die trotz entsprechender Kinematikkriterien der S3-Leitlinie nicht medizinisch vorstellig wurden. In mehreren Fällen berichtete der Notarzt von unverletzten Fahrzeuginsassen und/oder einer durch den Patienten abgelehnten Einweisung. Die Erfassung aller Beteiligten würde die Rate an Übertriage weiter erhöhen.

- Nicht in allen Fällen lag eine vollständige Dokumentation des Notarztes bzw. Rettungsdiensts vor.

- Die dokumentierte Geschwindigkeitsveränderung basierte ausnahmslos auf der Annahme durch Notarzt, Rettungsdienst oder Polizei bzw. auf Angaben der Patienten.

- Nicht berücksichtigt wurden unfallspezifische Daten wie das Vorhandensein und Auslösen von Airbags, die Anlage des Sicherheitsgurts, die Fahrzeugkonstruktion mit Knautschzone oder Alter des Fahrzeugs und der darin enthaltenen Sicherheitsausstattung. Ebenfalls wurde die Position des Patienten im Fahrzeug zum Unfallzeitpunkt nicht berücksichtigt.

- Bereits seit Jahren wird der ISS bezüglich seiner Aussagekraft bei der Einschätzung der Verletzungsschwere diskutiert [15, 26]. Zum Vergleich mit der gängigen Literatur wird der Vergleich in dieser Arbeit dennoch aufgeführt.

\section{Ausblick}

Die Ergebnisse dieser Studie sollten als Anlass zu weiteren Untersuchungen im Sinne einer mehrjährigen multizentrischen Studie genutzt werden. Zur Erfassung der tatsächlich während des Unfalls wirkenden Kräfte, Geschwindigkeitsveränderung etc. sollten, soweit bei modernen Fahrzeugen vorhanden, Unfalldatenschreiber ausgewertet und deren Telemetriedaten verwendet werden. Vorstellbar wäre es diese Telemetriedaten bereits im Zuge eines automatisierten Notrufs (eCall) an die Rettungsleitstelle zu übermitteln, um eine entsprechende Alarmierung von Einsatzkräften zu optimieren.

Auch wäre eine Auswertung der Verletzungsmuster nach Körperregion, Schwere sowie Therapieregime denkbar.

Durch das Anpassen der bisher gültigen Handlungsempfehlungen zu den Kriterien des Unfallmechanismus wäre es möglich, die Übertriage bei erhaltener Patientensicherheit zu reduzieren. Dies würde zu einer erheblichen Entlastung notfallmedizinischer Ressourcen führen und damit den Warte- und Behandlungszeiten aller Patienten zugutekommen.

\section{Fazit für die Praxis}

Unfallhergang und -geschwindigkeit bei Pkw/Lkw-Unfällen alleine sind kein sicherer Prädiktor für die Verletzungsschwere eines Patienten und sollten daher kritisch bezüglich der Initiierung einer Schockraumversorgung hinterfragt werden.

\section{Korrespondenzadresse}

\section{Prof. Dr. med. E. Popp}

Klinik für Anästhesiologie, Sektion

Notfallmedizin, Universitätsklinikum

Heidelberg

Im Neuenheimer Feld 110, 69120 Heidelberg,

Deutschland

Erik.Popp@uni-heidelberg.de

Funding. Open Access funding provided by Projekt DEAL.

\section{Einhaltung ethischer Richtlinien}

Interessenkonflikt. J.C. Stephan, T.Grossner, L.M.Stephan-Paulsen, M.A. Weigand, G. Schmidmaier und E. Popp geben an, dass kein Interessenkonflikt besteht.

Alle beschriebenen Daten wurden mit Zustimmung der zuständigen Ethik-Kommission, im Einklang mit nationalem Recht sowie gemäß der Deklaration von Helsinki von 1975 (in der aktuellen, überarbeiteten Fassung) erhoben.

Open Access. Dieser Artikel wird unter der Creative Commons Namensnennung 4.0 International Lizenz veröffentlicht, welche die Nutzung, Vervielfältigung, Bearbeitung, Verbreitung und Wiedergabe in jeglichem Medium und Format erlaubt, sofern Sie den/die ursprünglichen Autor(en) und die Quelle ordnungsgemäß nennen, einen Link zur Creative Commons Lizenz beifügen und angeben, ob Änderungen vorgenommen wurden.

Die in diesem Artikel enthaltenen Bilder und sonstiges Drittmaterial unterliegen ebenfalls der genannten Creative Commons Lizenz, sofern sich aus der Abbildungslegende nichts anderes ergibt. Sofern das betreffende Material nicht unter der genannten Creative Commons Lizenz steht und die betreffende Handlung nicht nach gesetzlichen Vorschriften erlaubt ist, ist für die oben aufgeführten Weiterverwendungen des Materials die Einwilligung des jeweiligen Rechteinhabers einzuholen.

Weitere Details zur Lizenz entnehmen Sie bitte der Lizenzinformation auf http://creativecommons.org/ licenses/by/4.0/deed.de.

\section{Literatur}

1. Statistisches Bundesamt (2016) Unfall, Gewalt, Selbstverletzung; Ergebnisse der amtlichen Statistikzum Verletzungsgeschehen 2014. Wiesbaden

2. Deutsche Gesellschaft für Unfallchirurgie e.V. (2016) S3-Leitlinie Polytrauma/SchwerverletztenBehandlung, Berlin

3. MacKenzie EJ, Rivara FP, Jurkovich GJ, Nathens AB, Frey KP, Egleston BL, Salkever DS, Scharfstein DO (2006) A national evaluation of the effect of trauma-center care on mortality. N Engl J Med 354(4):366-378. https://doi.org/10.1056/ NEJMsa052049

4. Haas B, Gomez D, Zagorski B, Stukel TA, Rubenfeld GD, Nathens AB (2010) Survival of the fittest: the hidden cost of undertriage of major trauma. 
J Am Coll Surg 211(6):804-811. https://doi.org/10 1016/j.jamcollsurg.2010.08.014

5. Ernstberger A, Joeris A, DaigIM, Kiss M, Angerpointner K, Nerlich M, Schmucker U (2015) Decrease of morbidity in road traffic accidents in a high income country - an analysis of 24,405 accidents in a 21 year period. Injury 46:135-143. https://doi.org/10. 1016/S0020-1383(15)30033-4

6. Lackner CK, Burghofer K, Lazarovici M, Stolpe E, Mutschler WE (2007) Verletzungsmuster verunfallter PKW-Insassen mit Polytrauma. Notfall Rettungsmed 10(1):23-31. https://doi.org/10. 1007/s10049-006-0880-5

7. Kraftfahrtbundesamt (2014) Personenkraftwagen am 1. Januar 2014 nach ausgewählten Merkmalen. Tabellen, Teil 3. http:// www.kba.de/DE/Statistik/Fahrzeuge/Bestand/ Ueberblick/2014/2014_b_bestandsbarometer teil3_absolut.html?nn=1112446. Zugegriffen: 22 . Mai 2016

8. Marzi I, Lustenberger T, Störmann P, Mörs K Wagner N, Wutzler S (2019) Steigender Vorhalteaufwand für den Schockraum (Increasing overhead ressources of the trauma room). Unfallchirurg 122(1):53-58. https://doi.org/10.1007/ s00113-018-0484-9

9. Bieler D, Trentzsch H, Baacke M, Becker L, Düsing $\mathrm{H}_{\text {, }}$ Heind B, Jensen KO, Lefering R, Mand C, Özkurtul O Paffrath T, Schweigkofler U, Sprengel K, Wohlrath B, Waydhas C (2018) Optimization of criteria for activation of trauma teams: avoidance of overtriage and undertriage (Optimierung der Kriterien zur Schockraumalarmierung:Vermeidung von Überund Untertriage). Unfallchirurg 121(10):788-793. https://doi.org/10.1007/s00113-018-0553-0

10. Butcher NE, Balogh ZJ (2014) Update on the definition of polytrauma. Eur J Trauma Emerg Surg 40(2):107-111

11. Trentz O, Oestern HJ, Hempelmann G, Kolbow H, Sturm J, Trentz OA, Tscherne H (1978) Kriterien für die Operabilität von Polytraumatisierten (Criteria for the operability of patients with multiple injuries (author's transl)). Unfallheilkunde 81(6):451-458

12. Trentz O, Keel MJB (2012) Management schwer verletzter Patienten. In: Largiadèr F, Saeger HD Keel M (Hrsg) Checkliste Chirurgie. Thieme, Stuttgart, NewYork, Delhi

13. Deutsche Gesellschaft für Unfallchirurgie e. V. (2012) Weißbuch Schwerverletztenversorgung. Thieme, Berlin

14. Pape $H C$, Lefering $R$, Butcher $N$, Peitzman $A$, Leenen $L$, Marzi I, Lichte $P$, Josten $C$, Bouillon $B$, Schmucker U, Stahel P, Giannoudis P, Balogh Z (2014) The definition of polytrauma revisited: an international consensus process and proposal of the new 'Berlin definition'. J Trauma Acute Care Surg 77(5):780-786. https://doi.org/10.1097/TA 0000000000000453

15. Fani-Salek MH, Totten VY, Terezakis SA (1999) Trauma scoring systems explained. Emerg Med Australas 11(3):155-166. https://doi.org/10.1046/ j.1442-2026.1999.00039.x

16. Newgard CD, Zive D, Holmes JF, Bulger EM, Staudenmayer K, Liao M, Rea T, Hsia RY, Wang NE, Fleischman R, Jui J, Mann NC, Haukoos JS, Sporer KA, Gubler KD, Hedges JR (2011) A multisite assessment of the American College of Surgeons Committee on Trauma field triage decision scheme for identifying seriously injured children and adults. J Am Coll Surg 213(6):709-721. https://doi. org/10.1016/j.jamcollsurg.2011.09.012

17. Santaniello JM, Esposito TJ, Luchette FA, Atkian DK, Davis KA, Gamelli RL (2003) Mechanism of injury doesnotpredictacuity or level of service need:field triage criteria revisited. Surgery 134(4):698-703. https://doi.org/10.1016/S0039

18. Brown JB, Stassen NA, Bankey PE, Sangosanya AT, Cheng JD, Gestring ML (2011) Mechanism of injury and special consideration criteria still matter: an evaluation of the National Trauma Triage Protocol. J Trauma 70(1):38-44. https://doi.org/10.1097/TA. 0b013e3182077ea8

19. Lerner EB, Shah MN, Cushman JT, Swor RA, Guse CE, Brasel K, Blatt A, Jurkovich GJ (2011) Does mechanism of injury predict trauma center need? Prehosp Emerg Care 15(4):518-525. https://doi. org/10.3109/10903127.2011.598617

20. Frink M, Lechler P, Debus F, Ruchholtz S (2017) Multiple Trauma and Emergency Room Management. Dtsch Arztebl Int 114(29-30):497-503

21. Huber-Wagner $S$, Lefering R, Qvick LM, Körner $M$ Kay MV, Pfeifer KJ, Reiser M, Mutschler W, Kanz KG (2009) Effect of whole-body CT during trauma resuscitation on survival: a retrospective, multicentre study. Lancet 373(9673):1455-1461. https://doi.org/10.1016/S0140-6736(09)60232-4

22. Kloth JK, Kauczor HU, Hosch W (2011) Bildgebung im Schockraum. Med Klin Intensivmed Notfallmed 106(2):82

23. Brenner DJ, Elliston CD (2004) Estimated radiation risks potentially associated with full-body CT screening. Radiology 232(3):735-738

24. Newgard CD, Staudenmayer K, Hsia RY, Mann NC, Bulger EM, Holmes JF, Fleischman R, Gorman K, Haukoos J, McConnell KJ (2013) The cost of overtriage: more than one-third of low-risk injured patients were taken to major trauma centers. Health Aff 32(9):1591-1599. https://doi.org/10. 1377/hlthaff.2012.1142

25. Lefering R, Paffrath T, NienaberU (2015) TraumaRegister DGU ${ }^{\circledR}$ Jahresbericht 2015. für den Zeitraum bis Ende 2014

26. Henry MC, Alicandro JM, Hollander JE, Moldashel JG, Cassara G, Thode HC (1996) Evaluation of American College of Surgeons trauma triage criteria in a suburban and rural setting. Am J Emerg Med 14(2):124-129. https://doi.org/10. 1016/S0735-6757(96)90117-5

27. Kononen DW, Flannagan CAC, Wang SC (2011) Identification and validation of a logistic regression model for predicting serious injuries associated with motor vehicle crashes. Accid Anal Prev 43(1):112-122

28. Spering C, Roessler M, Kurlemann T, Dresing K, Stürmer KM, Lehmann W, Sehmisch S (2017) Optimierte Ressourcenmobilisation und Versorgungsqualität Schwerstverletzter durch eine strukturierte Schockraumalarmierung (Optimized resource mobilization and quality of treatment of severely injured patients through a structured trauma room alarm system). Unfallchirurg. https:// doi.org/10.1007/s00113-017-0447-6

29. Lerner EB, Cushman JT, Blatt A, Lawrence RD, Shah MN, Swor RA, Brasel K, Jurkovich GJ (2011) EMS Provider assessment of vehicle damage compared with assessment by a professional crash reconstructionist. Prehosp Emerg Care 15(4):483-489

30. Sasser SM, Hunt RC, Sullivent EE, Wald MM, Mitchko J, Jurkovich GJ, Henry MC, Salomone JP, Wang SC, Galli RL, Cooper A, Brown LH, Sattin RW (2009) Guidelines forfield triage of injured patients. Recommendations of the National Expert Panel on Field Triage. Mmwr Morb Mortal Wkly Rep 58(RR1):1-35

31. Boyle MJ, Smith EC, Archer F (2008) Is mechanism of injury alone a useful predictor of major trauma? Injury 39(9):986-992. https://doi.org/10.1016/j. injury.2008.03.015

\section{Telemedizin}

Marx, Gernot, Rossaint, Rolf, Marx, Nikolaus (Hrsg.)

2021, XVI, 502 S. 98 Abb., 79,99 EUR ISBN 978-3-662-60610-0

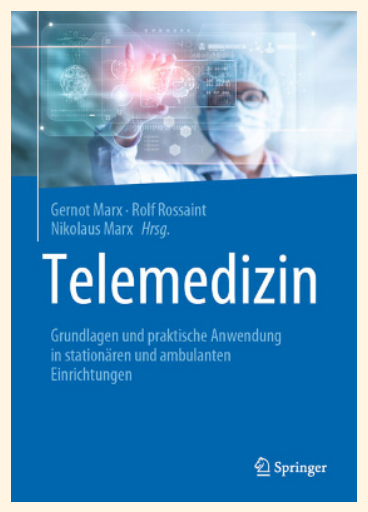

Grundlagen und praktische Anwendung in stationären und ambulanten Einrichtungen:

- Chancen, Risiken und rechtliche Besonderheiten der Telemedizin

- Mit zahlreichen Anwendungsbeispielen

- Mit Blick in die EU und Nachbarländer

Das Buch gibt einen umfassenden Überblick über den aktuellen Stand und die Entwicklungen im Bereich der Telemedizin in Deutschland. Das Herausgeberteam aus einem der führenden Telemedizinzentren Deutschlands und ein interdisziplinäres Autorenteam liefern wesentliche Grundlagen sowie zahlreiche Anwendungsbeispiele aus dem stationären und ambulanten Bereich. Zudem werden Chancen und Risiken der Telemedizin erörtert, so dass der Leser gut für die Zukunft in Klinik oder Praxis gerüstet ist. Ein Grundlagen- und Nachschlagewerk für alle Ärzte, die sich für digitale Zukunft im Gesundheitswesen wappnen und ihre Patienten optimal versorgen möchten. 